K. Miyake

Nagoya Math. J.

Vol. 120 (1990), 67-76

\title{
LEOPOLDT KERNELS AND CENTRAL EXTENSIONS OF ALGEBRAIC NUMBER FIELDS
}

\author{
KATSUYA MIYAKE
}

To Professor Michio Kuga

\section{$\S 1$. Introduction}

Let $k$ be an algebraic number field of finite degree, and $p$ be a fixed rational prime. We denote the set of all the non-Archimedian prime divisors of $k$ by $S_{0}(k)$ and the set of all the real Archimedian ones by $S_{\infty}^{+}(k)$. Put $S_{0}=\left\{\mathfrak{p} \in S_{0}(k)|\mathfrak{p}| p\right\}, S_{\infty}=S_{\infty}^{+}(k)$ and $S=S_{0} \cup S_{\infty}$, and define a subgroup of the unit group $\mathcal{O}^{\times}(k)$ of $k$ by

$$
E(k ; S)=\left\{\varepsilon \in \mathcal{O}^{\times}(k) \mid \varepsilon \equiv 1 \bmod \mathfrak{p} \text { for } \mathfrak{p} \in S\right\} .
$$

Then the $Z_{p}$-module $\bar{E}^{(p)}(k):=E(k ; S) \otimes_{Z} Z_{p}$ is isomorphic to $\mu_{p}(k) \times Z_{p}^{r}$, or to $Z_{p}^{r}$ if $p=2$ and $r_{1}>0$ where $\mu_{p}(k)$ is the multiplicative group of the $p$-power roots of 1 in $k$ and $r=r_{1}+r_{2}-1, r_{1}=\left|S_{\infty}^{+}(k)\right|, 2 r_{2}=[k: Q]-r_{1}$, is the essential $Z$-rank of $\mathcal{O}^{\times}(k)$. Furthermore we can define a homomorphism $\pi_{S}: \bar{E}^{(p)}(k) \rightarrow \prod_{\mathfrak{p} \in S_{0}} k_{\mathfrak{p}}^{\times}$by the diagonal embedding of $k^{\times}$into the last direct product. The kernel of $\pi_{S}$ is the Leopoldt kernel for $k, p$ and $S$, and denoted by $\mathscr{L}_{p}(k)$ or by $\mathscr{L}^{(p)}(k ; S)$.

The Leopoldt conjecture is to claim $\mathscr{L}_{p}(k)=1$, and known to be true for every $p$ in case where $k$ is a subfield of not only abelian extensions but also certain types of extensions containing non-abelian 2-extensions of imaginary quadratic fields, and also of some special extensions of $\boldsymbol{Q}$ (e.g. Brumer [B], Miyake [M1] etc.)

It is known by Nguyen-Quang-Do [Ng], Heider [H2] and others that $\mathscr{L}_{p}(k)$ is isomorphic to the Pontrjagin dual of the Schur multiplier of the group of a certain Galois extension. More precisely speaking, let $k^{(p)}(S)$ be the maximal $p$-extension of $k$ unramified outside $S$. Then we have

$$
H^{2}\left(\operatorname{Gal}\left(k^{(p)}(S) / k\right), \boldsymbol{Q} / Z\right) \cong \operatorname{Hom}\left(\mathscr{L}_{p}(k), \boldsymbol{Q} / Z\right) .
$$

Received September 22, 1988. 
One of our purposes of this paper is to clear the meaning of this isomorphism. Let $k^{(p)}$ be the maximal $p$-extension of $k$, and $M$ be the maximal central $p$-extension of $k^{(p)}(S) / k$. Then the maximal abelian $p$-extension $k^{\mathrm{ab}(p)}$ of $k$ is a subfield of $M$. Put $\mathbb{S}^{(p)}=\mathrm{Gal}\left(k^{(p)} / k\right), \mathrm{g}^{(p)}=\mathrm{Gal}\left(k^{(p)}(S) / k\right)$ and $\mathfrak{n}^{(p)}=\operatorname{Gal}\left(k^{(p)} / k^{(p)}(S)\right)$. Then we have

$$
H^{2}\left(\mho^{(p)}, \boldsymbol{Q} / Z\right)=0
$$

by Tate [Se], and easily see

$$
H^{2}\left(\mathrm{~g}^{(p)}, \boldsymbol{Q} / Z\right) \cong \operatorname{Hom}\left(\operatorname{Gal}\left(M / k^{(p)}(S) \cdot k^{\mathrm{ab}(p)}\right), \boldsymbol{Q} / Z\right)
$$

by the Hochschild-Serre exact sequence for the short exact sequence

$$
1 \longrightarrow \mathfrak{n}^{(p)} \longrightarrow \mathbb{S S}^{(p)} \longrightarrow \mathrm{g}^{(p)} \longrightarrow 1 \text {. }
$$

As the duals to the same $H^{2}\left(\mathfrak{g}^{(p)}, \boldsymbol{Q} / \boldsymbol{Z}\right)$, therefore, $\mathscr{L}_{p}(k)$ is isomorphic to $\operatorname{Gal}\left(M / k^{(p)}(S) \cdot k^{\mathrm{ab}(p)}\right)$. We will establish a natural isomorphism between them directly by class field theory.

Here we have explained the special case of $S$ where $\mathscr{L}^{(p)}(k ; S)=\mathscr{L}_{p}(k)$ corresponds to the Leopoldt conjecture. We will treat a general set of prime divisors of $k$ as $S$ in what follows.

\section{§2. Leopoldt kernels}

Let $k$ and $p$ be as above. We denote the idele group of $k$ by $k_{A}^{\times}$. We consider $k^{\times}$a diagonally embedded subgroup of $k_{\boldsymbol{A}}^{\times}$.

Let $S$ be a set of prime divisors of $k$, and $S=S_{0} \cup S_{\infty}, S_{0}=S \cap S_{0}(k)$, $S_{\infty}=S-S_{0}$. Put

$$
E\left(k ; S_{\infty}\right)=\left\{\varepsilon \in \mathcal{O}^{\times}(k) \mid \varepsilon \equiv 1 \bmod \mathfrak{p} \text { for } \mathfrak{p} \in S_{\infty}\right\} ;
$$

this depends only upon $S_{\infty}^{+}:=S_{\infty} \cap S_{\infty}^{+}(k)$. Denote the topological closure of the projection of $E\left(k ; S_{\infty}\right)$ to the non-Archimedian part of $k_{\boldsymbol{A}}^{\times}$by $\bar{E}_{0}\left(k ; S_{\infty}\right)$; this is an abelian profinite group; let $\bar{E}_{0}^{(p)}\left(k ; S_{\infty}\right)$ be its $p$-primary part. The ring of $p$-adic integers $Z_{p}$ naturally acts on this group. It is well known that $\bar{E}_{0}^{(p)}\left(k ; S_{\infty}\right)$ is isomorphic to $\mu_{p}(k) \times Z_{p}^{r}$ or to $Z_{p}^{r}$ (in nontrivial cases for $p=2$ ) as $Z_{p}$-modules (cf. Chevalley [Ch] or Heider [H1], Satz 4, for example).

Let $\pi_{S_{0}}$ be the projection homomorphism of $k_{\boldsymbol{A}}^{\times}$onto its $S_{0}$-part, $\prod_{p \in S_{0}}^{\prime} k_{p}^{\times}$, where $k_{\mathfrak{p}}$ is the completion of $k$ by $\mathfrak{p}$ and $\Pi^{\prime}$ denotes the restricted product with respect to the maximal compact subgroup $\mathcal{O}^{\times}\left(k_{\mathfrak{p}}\right)$ of $k_{\mathfrak{p}}^{\times}$for $\mathfrak{p} \in S_{0}$. 
The groups

$$
\mathscr{L}(k ; S):=\operatorname{Ker} \pi_{S_{0}} \cap \bar{E}_{0}\left(k ; S_{\infty}\right),
$$

and

$$
\mathscr{L}^{(p)}(k ; S):=\operatorname{Ker} \pi_{S_{0}} \cap \bar{E}_{0}^{(p)}\left(k ; S_{\infty}\right)
$$

are the Leopoldt kernels in $k$ for $S$ and $p$. It is not difficult to see that this $\mathscr{L}^{(p)}(k ; S)$ for $S=S_{0} \cup S_{\infty}$ with $S_{0}=\left\{p \in S_{0}(k)|\mathfrak{p}| p\right\}$ and $S_{\infty}=S_{\infty}^{+}(k)$ is canonically isomorphic to $\mathscr{L}_{p}(k)$ which was given in the preceding section; hence we also denote $\mathscr{L}^{(p)}(k ; S)$ in this case by $\mathscr{L}_{p}(k)$ for simplicity.

The homomorphism $\pi_{s_{0}}$ maps the torsion part $\left(\cong \mu_{p}(k)\right.$ if exists) of $\bar{E}_{0}^{(p)}\left(k ; S_{\infty}\right)$ injectively if $S_{0} \neq \phi$; then $\mathscr{L}^{(p)}(k ; S)$ is a torsion free $Z_{p}$-module.

The abelian pro-p-group $\bar{E}_{0}^{(p)}\left(k ; S_{\infty}\right)$ is mapped into the abelian profinite group $\prod_{\mathfrak{p} \in S_{0}} \mathcal{O}^{\times}\left(k_{\mathfrak{p}}\right)$ by $\pi_{S_{0}}$. If $\mathfrak{p}$ does not divide $p$, the $p$-primary part of $\mathcal{O}^{\times}\left(k_{\mathrm{p}}\right)$ is equal to the finite cyclic group $\mu_{p}\left(k_{\mathrm{p}}\right)$ of $p$-power roots of 1 in $k_{p}$. Therefore the $p$-primary part of the direct product $\prod \mathcal{O}^{\times}\left(k_{\mathfrak{p}}\right)$ is a finite group if $\mathfrak{p} \nmid p$ for every $\mathfrak{p} \in S_{0}$ and if $\left|S_{0}\right|<+\infty$; then the $Z_{p}$-rank of $\mathscr{L}^{(p)}(k ; S)$ coincides the essential $Z_{p}$-rank of $\bar{E}_{0}^{(p)}\left(k ; S_{\infty}\right)$ and is equal to $r=r_{1}+r_{2}-1$. Summing up, we have

Proposition 1. (1) If $S_{0} \neq \phi$, then $\mathscr{L}^{(p)}(k ; S)$ is a torsion free $Z_{p^{-}}$ module.

(2) If $0<\left|S_{0}\right|<+\infty$, and if $\mathfrak{p} \nmid p$ for every $\mathfrak{p} \in S_{0}$, then the $Z_{p}$-rank of $\mathscr{L}^{(p)}(k ; S)$ is equal to the essential Z-rank of $\mathcal{O}^{\times}(k)$; hence in particular, the quotient group $\bar{E}_{0}^{(p)}\left(k ; S_{\infty}\right) / \mathscr{L}^{(p)}(k ; S)$ is a finite p-group in this case.

Remark. If $p \neq 2$, then $\bar{E}_{0}^{(p)}\left(k ; S_{\infty}\right)=\bar{E}_{0}^{(p)}(k ; \phi)$ because the quotient group $E(k ; \phi) / E\left(k ; S_{\infty}\right)$ is an elementary 2-group.

\section{$\S 3$. The central extensions}

For a given set $S$ of prime divisors of $k$, we denote the maximal $p$ extension (abelian $p$-extension or abelian extension, resp.) of $k$ which is unramified outside $S$ by $k^{(p)}(S)\left(k^{\mathrm{ab}(p)}(S)\right.$ or $k^{\mathrm{ab}}(S)$, resp.).

Let $S_{\infty}$ be the Archimedian part of $S$ as above, and put $\bar{S}_{\infty}:=S_{0}(k) \cup S_{\infty}$. Then by definition, $\operatorname{Gal}\left(k^{\mathrm{ab}} / k^{\mathrm{ab}}\left(\bar{S}_{\infty}\right)\right)$ is a (finite) elementary 2-group. If $p \neq 2$, therefore, we have $k^{\mathrm{ab}(p)}\left(\bar{S}_{\infty}\right)=k^{\mathrm{ab}(p)}$.

Suppose that a finite Galois extension $K / k$ is unramified outside $S$. Let $S^{K}$ be the set of all the prime divisors of elements of $S$ in $K$. If $K / k$ is a $p$-extension, then the maximal $p$-extension of $K$ unramified outside 
$S^{K}$ is none other than $k^{(p)}(S)$. We simply write $K^{\mathrm{ab}(p)}(S)$ for $K^{\mathrm{ab}(p)}\left(S^{K}\right)$, etc., because there is no fears of ambiguity.

In general, we denote the maximal central extension (or $p$-extension, resp.) of a Galois extension $F / k$ by $(F / k)^{\operatorname{cen}}$ (or $(F / k)^{\operatorname{cen}(p)}$, resp.).

TheOREm 1. Let the notation and the assumptions be as above. Then the Galois groups of the extensions

$$
\left(K^{\mathrm{ab}}(S) / k\right)^{\mathrm{cen}} \cap K^{\mathrm{ab}}\left(\bar{S}_{\infty}\right) / K^{\mathrm{ab}}(S) \cdot k^{\mathrm{ab}}\left(\bar{S}_{\infty}\right)
$$

and

$$
\left(K^{\mathrm{ab}}(S) / k\right)^{\mathrm{cen}} \cap K^{\mathrm{ab}(p)}\left(\overline{\mathrm{S}}_{\infty}\right) / K^{\mathrm{ab}(p)}(S) \cdot k^{\mathrm{ab}(p)}\left(\bar{S}_{\infty}\right)
$$

are naturally isomorphic to the quotient groups

$$
\mathscr{L}(k ; S) / N_{K / k} \mathscr{L}\left(K ; S^{K}\right)
$$

and

$$
\mathscr{L}^{(p)}(k ; S) / N_{K / k} \mathscr{L}^{(p)}\left(K ; S^{K}\right),
$$

respectively, where $N_{K / k}: K_{\boldsymbol{A}}^{\times} \rightarrow k_{\boldsymbol{A}}^{\times}$is the norm map of $K / k$.

Proof. Put $\mathfrak{g}=\operatorname{Gal}(K / k), \quad \mathscr{S}=\operatorname{Gal}\left(K^{\mathrm{ab}}\left(\bar{S}_{\infty}\right) / k\right), \quad \mathfrak{A}=\operatorname{Gal}\left(K^{\mathrm{ab}}\left(\overline{\boldsymbol{S}}_{\infty}\right) / K\right)$ and $\mathfrak{B}=\operatorname{Gal}\left(K^{\mathrm{ab}}\left(\bar{S}_{\infty}\right) / K^{\mathrm{ab}}(S)\right)$. Then $\mathfrak{X}$ and $\mathfrak{B}$ are abelian closed normal subgroups of $\mathbb{S}$, and $\mathfrak{g}=\mathbb{E} / \mathscr{T}$. The inner automorphisms of (\&) naturally determines an action of $\mathfrak{g}$ on $\mathfrak{A}$ and $\mathfrak{B}$. The subgroup [ㅁ, $\mathfrak{B}]$ of commutators of $\mathfrak{B}$ and $\mathbb{S}$ coincides with

$$
\mathfrak{B}^{\Delta_{g}}:=\left\langle a^{1-\sigma} \mid a \in \mathfrak{B}, \sigma \in \mathfrak{g}\right\rangle
$$

where the right hand side means the topologically generated closed subgroup of $\mathfrak{B}$. Therefore we have

$$
\operatorname{Gal}\left(K^{\mathrm{ab}}\left(\bar{S}_{\infty}\right) /\left(K^{\mathrm{ab}}(S) / k\right)^{\mathrm{cen}} \cap K^{\mathrm{ab}}\left(\bar{S}_{\infty}\right)\right)=\mathfrak{B}^{\lrcorner_{\mathrm{g}}} .
$$

Now let $\alpha_{K}: K_{A}^{\times} \rightarrow \operatorname{Gal}\left(K^{\mathrm{ab}} / K\right)$ be the Artin map of class field theory; this is a surjective homomorphism the kernel $K^{\#}$ of which is the closure of $K^{\times} \cdot K_{\infty+}^{\times}$in the idele group $K_{A}^{\times}$where $K_{\infty+}^{\times}$is the connected component of 1 in $K_{A}^{\times}$. Put $S_{\infty}^{\prime}=S_{\infty}^{+}(k)-S_{\infty}$ and

$$
K^{\sharp}\left(S_{\infty}^{\prime}\right):=K^{\sharp} \cdot \prod_{\Re \in S_{\infty}^{\prime K}} K_{\Re}^{\times} .
$$

Then $\operatorname{Gal}\left(K^{\mathrm{ab}} / K^{\mathrm{ab}}\left(\bar{S}_{\infty}\right)\right)$ is isomorphic to the elementary 2-group $K^{\sharp}\left(S_{\infty}^{\prime}\right) / K^{\sharp}$ by $\alpha_{K}$. 
Through the inner automorphisms of $\operatorname{Gal}\left(K^{\mathrm{ab}}\left(\overline{\boldsymbol{S}}_{\infty}\right) / k\right)$, the abelian group $\operatorname{Gal}\left(K^{\mathrm{ab}}\left(\overline{\boldsymbol{S}}_{\infty}\right) / K\right)$ becomes a $\mathrm{g}$-module; $\alpha_{K}$ induces a $\mathrm{g}$-isomorphism of $K_{A}^{\times} / K^{\sharp}\left(S_{\infty}^{\prime}\right)$ onto $\operatorname{Gal}\left(K^{\mathrm{ab}}\left(\bar{S}_{\infty}\right) / K\right)$ which maps the $p$-primary part $\left(K_{A}^{\times} / K^{\ddagger}\left(S_{\infty}^{\prime}\right)\right)^{(p)}$ onto $\mathfrak{Q}^{(p)}=\operatorname{Gal}\left(K^{\mathrm{ab}(p)}\left(\bar{S}_{\infty}\right) / K\right)$. Denote the subgroup of $K_{A}^{\times}$, $\left\{\left(\cdots, x_{\Re}, \cdots\right) \in \mathcal{O}^{\times}\left(K_{A}\right) \mid x_{\mathfrak{\beta}}=1\right.$ for $\mathfrak{\beta} \in S_{0}^{K}$ and for every Archimedian $\left.\mathfrak{\beta}\right\}$ by $U^{\prime}\left(K ; S_{0}\right)$. Then by $\alpha_{K}, \mathfrak{B}$ corresponds to

$$
U^{\prime}\left(K ; S_{0}\right) \cdot K^{\sharp}\left(S_{\infty}^{\prime}\right) / K^{\sharp}\left(S_{\infty}^{\prime}\right) .
$$

It is also known by class field theory that

$$
\operatorname{Gal}\left(K^{\mathrm{ab}}\left(\bar{S}_{\infty}\right) / K \cdot k^{\mathrm{ab}}\left(\overline{\boldsymbol{S}}_{\infty}\right)\right)
$$

corresponds to

$$
N_{\bar{K} / k}^{-1}\left(k^{\sharp}\left(S_{\infty}^{\prime}\right)\right) / K^{\sharp}\left(S_{\infty}^{\prime}\right)
$$

by $\alpha_{K}$ where $k^{\sharp}\left(S_{\infty}^{\prime}\right)=k^{\sharp} \cdot \prod_{p \in S_{\infty}^{\prime}} k_{p}^{\times}$and $k^{\sharp}$ is the kernel of the Artin map $\alpha_{k}$ of $k$. Therefore by $\alpha_{K}$,

$$
\operatorname{Gal}\left(\left(K^{\mathrm{ab}}(S) / k\right)^{\mathrm{cen}} \cap K^{\mathrm{ab}}\left(\overline{\mathrm{S}}_{\infty}\right) / K^{\mathrm{ab}}(S) \cdot k^{\mathrm{ab}}\left(\overline{\mathrm{S}}_{\infty}\right)\right)
$$

is isomorphic to

$$
\begin{aligned}
& U^{\prime}\left(K ; S_{0}\right) \cdot K^{\sharp}\left(S_{\infty}^{\prime}\right) \cap N_{K^{\prime} / k}^{-1}\left(k^{\sharp}\left(S_{\infty}^{\prime}\right)\right) / U^{\prime}\left(K ; S_{0}\right)^{d_{s}} \cdot K^{\sharp}\left(S_{\infty}^{\prime}\right) \\
& \quad \cong\left(U^{\prime}\left(K ; S_{0}\right) \cap N_{K / k}^{-1}\left(k^{\sharp}\left(S_{\infty}^{\prime}\right)\right)\right) \cdot K^{\sharp}\left(S_{\infty}^{\prime}\right) / U^{\prime}\left(K ; S_{0}\right)^{d_{s}} \cdot K^{\sharp}\left(S_{\infty}^{\prime}\right) \\
& \quad \cong U^{\prime}\left(K ; S_{0}\right) \cap N_{K / k}^{-1 / k}\left(k^{\sharp}\left(S_{\infty}^{\prime}\right)\right) / U^{\prime}\left(K ; S_{0}\right)^{d_{8}} \cdot\left(U^{\prime}\left(K ; S_{0}\right) \cap K^{\sharp}\left(S_{\infty}^{\prime}\right)\right) .
\end{aligned}
$$

Lemma. (1) $U^{\prime}\left(K ; S_{0}\right)^{\lrcorner_{\beta}}=U^{\prime}\left(K ; S_{0}\right) \cap N_{K / k}^{-1}(1)$.

(2) $\quad U^{\prime}\left(K ; S_{0}\right) \cap K^{\ddagger}\left(S_{\infty}^{\prime}\right)=\mathscr{L}(K ; S)$.

Proof. (1) Let $\mathfrak{p}$ be a non-Archimedian prime divisor of $k$. The $p$-part of $U^{\prime}\left(K ; S_{0}\right)$ is trivial if $\mathfrak{p} \in S_{0}$ by definition. Suppose that $\mathfrak{p} \notin S_{0}$. Then $\mathfrak{p}$ is unramified in $K / k$. Therefore $K_{\mathfrak{q}} / k_{\mathfrak{p}}$ is cyclic for $\mathfrak{p} \mid \mathfrak{p}$. The $\mathfrak{p}$ part of $U^{\prime}\left(K ; S_{0}\right)$ is $\mathcal{O}^{\times}\left(K \otimes_{k} k_{\mathfrak{p}}\right)$. Fix a prime divisor $\mathfrak{P}$ of $\mathfrak{p}$ in $K$ and denote the norm map of the local extension $K_{\mathfrak{p}} / k_{\mathfrak{p}}$ by $N_{\mathfrak{p}}$. Then it is clear that

$$
N_{K / k}^{-1}(1) \cap \mathcal{O}^{\times}\left(K \underset{k}{\otimes} k_{\mathfrak{p}}\right)=\mathcal{O}^{\times}\left(K \otimes k_{\mathrm{p}}\right)^{\Lambda_{\mathfrak{q}}} \cdot\left(N_{\mathfrak{p}}^{-1}(1) \cap \mathcal{O}^{\times}\left(K_{\mathfrak{p}}\right)\right) .
$$

Since $K_{\mathfrak{p}} / k_{\mathfrak{p}}$ is unramified, we have

$$
N_{\mathfrak{p}}^{-1}(1) \cap \mathcal{O}^{\times}\left(K_{\mathfrak{p}}\right)=\mathcal{O}^{\times}\left(K_{\mathfrak{p}}\right)^{\mathcal{A}_{\mathfrak{B}}(\mathfrak{p})}
$$


where $\mathfrak{g}(\mathfrak{p})=\operatorname{Gal}\left(K_{\mathfrak{p}} / k_{\mathfrak{p}}\right) \smile \mathfrak{g}(\operatorname{cf}$. Iyanaga [I], Ch V, Th, 1.2). This shows that

$$
N_{K / k}^{-1}(1) \cap \mathcal{O}^{\times}\left(K \otimes k_{\mathfrak{p}}\right)=\mathcal{O}^{\times}\left(K \otimes k_{\mathfrak{p}}\right)^{A_{\mathfrak{q}}}
$$

for every $\mathfrak{p} \notin S_{0}$, and proves (1).

(2) By Shimura [Sh], 2.2, we easily see that

$$
K^{\sharp}\left(S_{\infty}^{\prime}\right)=\bar{E}_{0}\left(K ; S_{\infty}^{K}\right) \cdot K^{\times} \cdot\left(K_{\infty+}^{\times} \cdot \prod_{\mathfrak{R} \in S_{\infty}^{\prime K}} K_{p}^{\times}\right) .
$$

Suppose that $x$ is an element $U^{\prime}\left(K ; S_{0}\right) \cap K^{\sharp}\left(S_{\infty}^{\prime}\right)$ and $x=e \cdot a \cdot u, e \in \bar{E}_{0}\left(K ; S_{\infty}^{K}\right)$, $a \in K^{\times}, u \in\left(K_{\infty+}^{\times} \cdot \prod K_{p}^{\times}\right)$. Let $a=a_{0} \cdot a_{\infty}$ be the decomposition of $a$ in $K_{\boldsymbol{A}}^{\times}$ into its non-Archimedian part $a_{0}$ and its Archimedian part $a_{\infty}$. Then $a_{\infty} \cdot u=1$, and $x=e \cdot a_{0} \in U^{\prime}\left(K ; S_{0}\right)$. Hence $a$ is a unit of $K$ such that $a \equiv u^{-1} \equiv 1 \bmod \mathfrak{R}$ for every $\mathfrak{B} \in S_{\infty}^{K}$, i.e. $a \in E\left(K ; S_{\infty}^{K}\right)$. Therefore we have

$$
x=e \cdot a_{0} \in \bar{E}_{0}\left(K ; S_{\infty}^{K}\right) \cap U^{\prime}\left(K ; S_{0}\right)=\mathscr{L}(K ; S),
$$

and proved that the left hand side of (2) lies in the right hand side. The converse inclusion is clear. The proof of the lemma is completed.

By this lemma, we see that the Galois group $(*)$ is isomorphic to the quotient group,

$$
U^{\prime}\left(K ; S_{0}\right) \cap N_{K / k}^{-1}\left(k^{\sharp}\left(S_{\infty}^{\prime}\right)\right) / \mathscr{L}(K ; S) \cdot\left(U^{\prime}\left(K ; S_{0}\right) \cap N_{K / k}^{-1}(1)\right) .
$$

This is isomorphically mapped by $N_{K / k}$ onto

$$
N_{K / k}\left(U^{\prime}\left(K ; S_{0}\right)\right) \cap k^{\sharp}\left(S_{\infty}^{\prime}\right) / N_{K / k}(\mathscr{L}(K ; S)) .
$$

Since a non-Archimedian prime $\mathfrak{p} \notin S_{0}$ is unramified in $K / k$, we have

$$
N_{K / k}\left(U^{\prime}\left(K ; S_{0}\right)\right)=U^{\prime}\left(k ; S_{0}\right) .
$$

By (2) of the above lemma for $k$ in place of $K$, we have

$$
U^{\prime}\left(k ; S_{0}\right) \cap k^{\sharp}\left(S_{\infty}^{\prime}\right)=\mathscr{L}(k ; S) .
$$

The proof of Theorem 1 is now completed by taking the p-primary parts.

\section{$\S 4$. The main theorem}

The maximal nilpotent extension of $k$ which is unramified outside $S$ is denoted by $k^{\text {nil }}(S)$.

TheOREM 2. There exist surjective homomorphisms,

$$
\beta_{k, S}: \mathscr{L}(k ; S) \longrightarrow \operatorname{Gal}\left(\left(k^{\mathrm{ni1}}(S) / k\right)^{\operatorname{cen}} \cap k^{\mathrm{ni1}}\left(\bar{S}_{\infty}\right) / k^{\mathrm{ni1}}(S) \cdot k^{\mathrm{ab}}\left(\bar{S}_{\infty}\right)\right),
$$


and

$$
\beta_{k, S}^{(p)}: \mathscr{L}^{(p)}(k ; S) \longrightarrow \operatorname{Gal}\left(\left(k^{(p)}(S) / k\right)^{\operatorname{cen}} \cap k^{(p)}\left(\bar{S}_{\infty}\right) / k^{(p)}(S) \cdot k^{\mathrm{ab}(p)}\left(\bar{S}_{\infty}\right)\right)
$$

the kernels of which are, respectively,

$$
\operatorname{Ker} \beta_{k, S}=\bigcap_{\substack{k \subset K<k^{n i 1} \\[K: k]<+\infty}} N_{K / k}(\mathscr{L}(K ; S)),
$$

and

$$
\operatorname{Ker} \beta_{k, S}^{(p)}=\bigcap_{\substack{k \subset K<k^{(p)}(S) \\[K: k]<+\infty}} N_{K / k}\left(\mathscr{L}^{(p)}(K ; S)\right) .
$$

Furthermore, the following diagram is commutative for every finite Galois subextension $K / k$ of $k^{(p)}(S) / k$ :

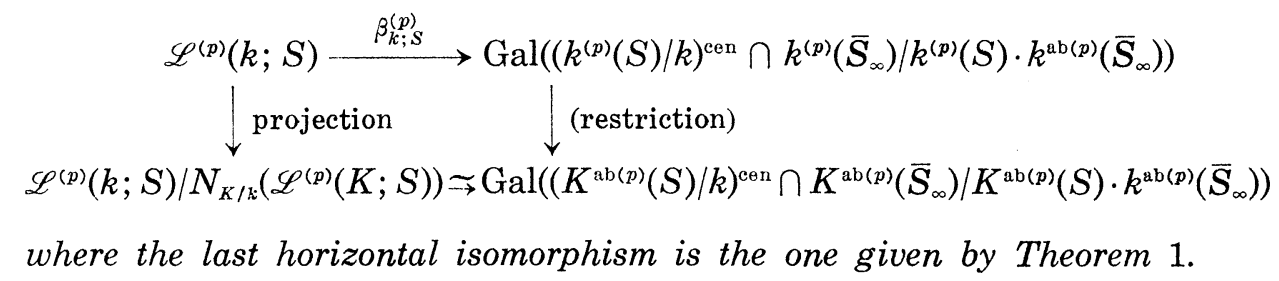

Proof. It is sufficient to show the $p$-case. (However it is to be noted that $K^{\mathrm{ab}}(S) / k$ and $\left(K^{\mathrm{ab}}(S) / k\right)^{\text {een }}$ are not nilpotent extensions in general even if $K / k$ is a $p$-extension.) Let $K / k$ be a finite Galois subextension of $k^{(p)}(S) / k$. Then it is clear that

$$
\left(K^{\mathrm{ab}(p)}(S) / k\right)^{\mathrm{cen}} \cap K^{\mathrm{ab}(p)}\left(\overline{\mathrm{S}}_{\infty}\right) \subset\left(k^{(p)}(S) / k\right)^{\mathrm{cen}} \cap k^{(p)}\left(\overline{\mathrm{S}}_{\infty}\right)
$$

and that

$$
\begin{aligned}
& \left(K^{\mathrm{ab}(p)}(S) / k\right)^{\operatorname{cen}} \cap K^{\mathrm{ab}(p)}\left(\bar{S}_{\infty}\right) \cap\left(k^{(p)}(S) \cdot k^{\mathrm{ab}(p)}\left(\bar{S}_{\infty}\right)\right) \\
& \quad=\left(\left(K^{\mathrm{ab}(p)}(S) / k\right)^{\mathrm{cen}} \cap K^{\mathrm{ab}(p)}\left(\bar{S}_{\infty}\right) \cap k^{(p)}(S)\right) \cdot k^{\mathrm{ab}(p)}\left(\bar{S}_{\infty}\right) \\
& \quad=K^{\mathrm{ab}(p)}(S) \cdot k^{\mathrm{ab}(p)}\left(\bar{S}_{\infty}\right) .
\end{aligned}
$$

Therefore the natural homomorphism, (restriction) of the diagram (defined by taking the restrictions), is surjective.

Let $x$ be an element of the field $\left(k^{(p)}(S) / k\right)^{\text {cen }} \cap k^{(p)}\left(\bar{S}_{\infty}\right)$. Then $k(x)$ and its Galois closure $F$ over $k$ is a finite $p$-extension of $k$. Put $K=F \cap k^{(p)}(S)$. Since $F \cdot k^{(p)}(S)$ is an abelian extension of $k^{(p)}(S)$, it is clear that $F$ is contained in $(K / k)^{\operatorname{cen}} \cap K^{\mathrm{ab}(p)}\left(\bar{S}_{\infty}\right)$ and hence also in $\left(K^{\mathrm{ab}(p)}(S) / k\right)^{\mathrm{cen}} \cap K^{\mathrm{ab}(p)}\left(\overline{\boldsymbol{S}}_{\infty}\right)$. This shows that the field $\left(k^{(p)}(S) / k\right)^{\text {cen }} \cap k^{(p)}\left(\bar{S}_{\infty}\right)$ is the composition of all of $\left(K^{\mathrm{ab}(p)}(S) / k\right)^{\mathrm{cen}} \cap K^{\mathrm{ab}(p)}\left(\bar{S}_{\infty}\right)$ for finite Galois subextensions $K / k$ of $k^{(p)}(S) / k$. 
Take another finite Galois subextension $L / k$ of $k^{(p)}(S) / k$ and suppose that $L$ contains $K$. Using the notation introduced in the proof of Theorem 1 , denote the $p$-primary part of $(* *)$ by $X(K / k)$, and also

$$
\operatorname{Gal}\left(\left(K^{\mathrm{ab}(p)}(S) / k\right)^{\mathrm{een}} \cap K^{\mathrm{ab}(p)}\left(\bar{S}_{\infty}\right) / K^{\mathrm{ab}(p)}(S) \cdot k^{\mathrm{ab}(p)}\left({\overline{S_{\infty}}}_{\infty}\right)\right)
$$

by $G(K / k)$ for simplicity. The pro-p-group $G(K / k)$ is canonically isomorphic to the $p$-primary part of the abelian profinite group $(*)$ in the proof of Theorem 1. We have the following diagram by class field theory:

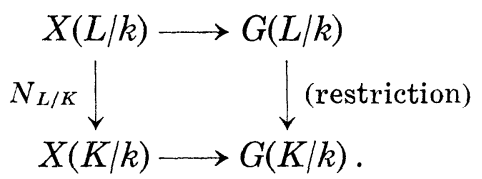

Since $N_{L / k}=N_{K / t} \circ N_{L / K}$, we have another commutative diagram,

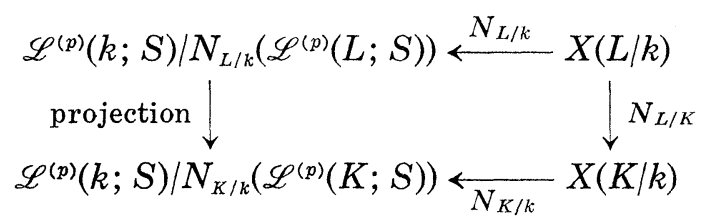

where the left vertical homomorphism is the projection which is naturally defined because

$$
N_{K / k}\left(\mathscr{L}^{(p)}(K ; S)\right) \supset N_{L / k}\left(\mathscr{L}^{(p)}(L ; S)\right)
$$

Combining these two diagrams, we have two isomorphic projective systems $\left\{\mathscr{L}^{(p)}(k ; S) / N_{K^{\prime} / k}\left(\mathscr{L}^{(p)}(K ; S)\right)\right\}$ and $\{G(K / k)\}$ both of which are parametrized by finite Galois subextensions $K / k$ of $k^{(p)}(S) / k$. We now see Theorem 2 at once if we take the projective limits.

Corollary (Heider [H 2], Satz 10). Suppose that the set $S$ contains all of the real Archimedian prime divisors of $k$, i.e. $S_{\infty} \supset S_{\infty}^{+}(k)$. Then the Schur multiplier $H^{2}\left(\operatorname{Gal}\left(k^{\mathrm{ni1}}(S) / k\right), \boldsymbol{Q} / Z\right)$ is dual to $\mathscr{L}(k ; S) / \operatorname{Ker} \beta_{k, s}$, and $H^{2}\left(\operatorname{Gal}\left(k^{(p)}(S) / k\right), \boldsymbol{Q} / Z\right)$ to $\mathscr{L}^{(p)}(k ; S) / \operatorname{Ker} \beta_{k, S}^{(p)}$ for every prime $p$.

Proof. By the assumption we have $k^{\mathrm{ni1}}\left(\bar{S}_{\infty}\right)=k^{\mathrm{nil}}$, the maximal nilpotent extension of $k$, and $k^{(p)}\left(\bar{S}_{\infty}\right)=k^{(p)}$. Therefore we see the corollary by Theorem 2 in a similar way to the one explained in Introduction using the result of Tate and the Hochschild-Serre exact sequence. 


\section{$\S 5$. The case related to the Leopoldt conjecture}

Here we take $S=S_{0} \cup S_{\infty}, S_{0}=\left\{p \in S_{0}(k)|p| p\right\}, S_{\infty}=S_{\infty}^{+}(k)$, and denote the Leopoldt kernel $\mathscr{L}^{(p)}(k ; S)$ simply by $\mathscr{L}_{p}(k)$.

Lemma (Heider [H2], Lemma 10). Let $k_{\infty}=\bigcup_{n=0}^{\infty} k_{n}$ be the cyclotomic $Z_{p}$-extension of $k=k_{0}$. Then we have

$$
\bigcap_{n=1}^{\infty} N_{k_{n} / k}\left(\mathscr{L}_{p}\left(k_{n}\right)\right)=1
$$

Proof. Heider [H 2] showed the case where a primitive $p$-th root $\zeta_{p}$ or $\sqrt{-1}$ if $p=2$ belongs to $k$. If not, put $k^{\prime}=k\left(\zeta_{p}\right)$ or $k(\sqrt{-1})$. Then the cyclotomic $Z_{p}$-extension $k_{\infty}^{\prime}=\bigcup_{m=0}^{\infty} k_{m}^{\prime}$ is the composite field $k^{\prime} \cdot k_{\infty}$. It is easy to see that

$$
N_{k_{n} / k}\left(\mathscr{L}_{p}\left(k_{n}\right)\right) \subset N_{k_{m}^{\prime} / k^{\prime}}\left(\mathscr{L}_{p}\left(k_{m}^{\prime}\right)\right)
$$

if $k_{m}^{\prime}=k^{\prime} \cdot k_{n}$. Therefore we have

$$
\bigcap_{n=1}^{\infty} N_{k_{n} / k}\left(\mathscr{L}_{p}\left(k_{n}\right)\right) \subset \bigcap_{m=1}^{\infty} N_{k_{m}^{\prime} / k^{\prime}}\left(\mathscr{L}_{p}\left(k_{m}^{\prime}\right)\right)=1 .
$$

Since the cyclotomic $Z_{p}$-extension $k_{\infty}$ of $k$ is contained in $k^{(p)}(S)$ in our case here, we have by Theorem 2 and the corollary to it at once

TheOREM 3. Let the notation and the assumptions be as above. There exists a natural isomorphism

$$
\beta_{k}^{(p)}: \mathscr{L}_{p}(k) \longrightarrow \operatorname{Gal}\left(\left(k^{(p)}(S) / k\right)^{\mathrm{cen}} \cap k^{(p)} / k^{(p)}(S) \cdot k^{\mathrm{ab}(p)}\right) .
$$

In particular, therefore, the Schur multiplier $H^{2}\left(\operatorname{Gal}\left(k^{(p)}(S) / k\right), \boldsymbol{Q} / \boldsymbol{Z}\right)$ is naturally dual to the Leopoldt kernel $\mathscr{L}_{p}(k)$.

\section{REFERENCES}

[B] A. Brumer, On the units of algebraic number fields, Mathematica, 14 (1967), 121-124.

[Ch] C. Chevalley, Deux théoremes d'arithmétique, J. Math. Soc. Japan, 3 (1951) , 36-44.

[H 1] F.-P. Heider, Zahlentheoretische Knoten unendlicher Erweiterungen, Arch. Math., 37 (1981), 341-352.

[H 2] - Kapitulationsproblem und Knotentheorie, Manusc. Math., 46 (1984), 229 272.

[I] S. Iyanaga (Ed.), The Theory of Numbers, North-Holland Publish. Company, Amsterdam. Oxford, 1975.

[M 1] K. Miyake, On the units of an algebraic number field, J. Math. Soc. Japan, 34 (1982), 516-525.

[M 2] - On central extensions of a Galois extension of algebraic number fields, 
Nagoya Math. J., 93 (1984), 133-148.

[Ng] Thong Nguyen-Quang-Do, Sur la structure galoisienne des corps locaux et la theorie d'Iwasawa, Comp. Math., 46 (1982), 85-119.

[Se] J.-P. Serre, Modular forms of weight one and Galois representations, in: Algebraic Number Fields, ed. by A. Fröhlich, Acad. Press, London-San Francisco, 1977.

[Sh] G. Shimura, On canonical models of arithmetic quotient of bounded symmetric domains II, Ann. of Math., 92 (1970), 528-549.

Department of Mathematics

College of General Education

Nagoya University

Nagoya 464-01

Japan 